\title{
Research of Inonotus obliquus Oligosaccharide in Prevention of Hyperlipidemia
}

\author{
Dawei Wu $\mathbb{D}$, Yanrong Zhang ${ }^{\mathbb{D}}$, Dawei Wang, Tingting Liu, Shanshan Zhang, \\ and Chenhe Yang
}

College of Food Science \& Engineering, Jilin Agricultural University, Changchun 130118, China

Correspondence should be addressed to Yanrong Zhang; zhangyanrong0044@jlau.edu.cn

Received 14 September 2021; Revised 14 November 2021; Accepted 19 November 2021; Published 30 November 2021

Academic Editor: Yong Sun

Copyright (C) 2021 Dawei Wu et al. This is an open access article distributed under the Creative Commons Attribution License, which permits unrestricted use, distribution, and reproduction in any medium, provided the original work is properly cited.

\begin{abstract}
In this study, hot water was used to extract Inonotus obliquus oligosaccharide. DEAE-cellulose and Sepharose G-200 were used to purify Inonotus obliquus oligosaccharide. Inonotus obliquus oligosaccharide IOP-2A was obtained. Its molecular weight Mw is about $1000 \mathrm{Da}$. The monosaccharide composition and molar ratio were glucose : xylose : galactose : mannose $=54.1: 13.6: 13.2$ : 6.7. In addition, it also contains a small amount of galactose, gluconic acid, rhamnose, and fucose. IOP-2A contained mainly $\beta$-glycosidic bonds. Among them, 1,4-glycosidic bonds accounted for 9.2\%, and 1,6-glycosidic bonds accounted for $85.1 \%$. Oligosaccharide macromolecules formed a layered structure. Mouse experiments showed that IOP-2A had the function of preventing hyperlipidemia. At the same time, IOP-2A had a certain protective effect on the liver and kidney. The mechanism of IOP-2A in preventing hyperlipidemia was obtained from the perspective of mouse intestinal flora.
\end{abstract}

\section{Introduction}

Hyperlipidemia is a disease whose blood lipid level exceeds the standard [1-3] and can cause atherosclerosis, cardiovascular disease, cerebrovascular disease, and other diseases [4-6]. It seriously threatens people's health. The etiology of hyperlipidemia is related to diet, mood, insufficient exercise, etc. At present, the treatment method is to use statins, fibrates, niacin, and other drugs. The price of these drugs is high, which increases people's economic burden. At the same time, hypolipidemic drugs have certain side effects [7-9] and have certain damage to liver, gastrointestinal, and muscle tissues. The hypolipidemic effect of functional components in natural food has attracted people's attention. The main characteristic of these substances is that they have no adverse effects on the human body and have a good hypolipidemic effect.

The activities of oligosaccharides were studied by many scholars. For example, Govindan et al. studied the antioxidant and protective effect of Sapium sebiferum oligosaccharides on alcoholic liver injury in rats [10], and there are also some reports on the study of fungal oligosaccharides. Yin et al. studied the immunomodulatory effect of edible mushroom oligosaccharides [11], and Zhang and others extensively studied the healthcare function of oligosaccharides and other components of edible fungi and the high-value utilization of edible fungi [12]. Liu et al. studied the structural characterization and immune enhancement activity of Hericium erinaceus oligosaccharide enzyme modification [13-15]. Du et al. studied the chemical analysis and antioxidant activity of fungal oligosaccharides [16, 17]. Inonotus obliquus, the scientific name of birch antler, is a fungus growing on birch trees, which is widely distributed in Northeast China. In recent years, the functional research on natural substances such as fungal oligosaccharides had been continued to increase such as antioxidation [18-20], antitumor [21-23], antiradiation [24-26], lowering blood lipid [27], regulating intestinal function [28], antivirus [29], and lowering blood sugar [30-32].

However, there are few reports on the effect of Inonotus obliquus oligosaccharide on reducing blood lipid, and there is no report on the function of Inonotus obliquus oligosaccharide in preventing hyperlipidemia. The extraction and purification of Inonotus obliquus oligosaccharide and the function of purified oligosaccharide to prevent hyperlipidemia were studied in this paper. 


\section{Materials and Methods}

2.1. Equipment and Reagents. Gas chromatography-mass spectrometer was manufactured by Agilent Co., Ltd., USA. Inonotus obliquus fruiting body was purchased from Weihe Forestry Bureau, Heilongjiang Province, China. DEAEcellulose was purchased from Shanghai Yuanye Biotechnology Co., Ltd. Sepharose G-200 was purchased from Biotech Co., Ltd. Anhydrous ethanol and sodium chloride were purchased from Beijing chemical plant. Phenol was purchased from Shanghai Sinopec Mitsui Co., Ltd. Concentrated sulfuric acid was purchased from the chemical reagent factory of Sinopharm Group. N-Butanol and chloroform were purchased from Tianjin No. 3 Chemical Reagent Factory. Hydrochloric acid and glucose were purchased from Sinopharm chemical reagent factory. Sodium hydroxide was purchased from Tianjin North Tianyi Chemical Reagent Factory. Total cholesterol (T-Cho) test kit, triglyceride (TG) test kit, low-density lipoprotein cholesterol (LDL-C) determination kit, high-density lipoprotein cholesterol (HDL-C) determination kit, total superoxide dismutase (T-SOD) determination kit, and malondialdehyde (MDA) determination kit were purchased from Nanjing Jiancheng Bioengineering Institute. High-fat feed and ordinary feed were purchased from Nanjing Shengmin Scientific Research Animal Farm. Feed standards comply with GB/t14924.9-2001 (This standard specified the determination method of routine nutrients in formula feed for experimental animals, that is, the determination method of water, crude protein, crude fat, crude fiber, crude ash, calcium, and total phosphorus in formula feed.).

KM mice, which were 35 days old, of SPF grade, and male are used, and the quantity is 60 . They were purchased from Fukang Biotechnology Co., Ltd. (license no. SCXK (Beijing) 2020-0004).

\subsection{Extraction and Purification of Inonotus obliquus} Oligosaccharide. The fruiting body powder of Inonotus obliquus was accurately weighed. First, fat was removed, and it was decolorized by ethanol refluxing for three times. Then, it was heated with a water bath and extracted with hot water. The rotating evaporation temperature of the extract was $58^{\circ} \mathrm{C}$, and the rotating speed was $30 \mathrm{r} / \mathrm{min}$. Absolute ethanol was added to the concentrated liquid to make the final concentration of ethanol $80 \%$. It was placed in a $4{ }^{\circ} \mathrm{C}$ refrigerator for $12 \mathrm{~h}$. Then, it was centrifuged at centrifugal acceleration $2158 \times \mathrm{g}$. The Sevage reagent was added to the oligosaccharide solution to deproteinize, and the dosage of Sevage reagent was $1 / 4$ of the volume of oligosaccharide solution. Sevage reagent was prepared with n-butanol: chloroform $=1: 4$, usually deproteinized three times, and lyophilized at $-55^{\circ} \mathrm{C}, 0.05 \mathrm{mbar}$, and $12 \mathrm{~h}$. The crude oligosaccharide of Inonotus obliquus was obtained.

2.3. Purification of Inonotus obliquus Oligosaccharide. Inonotus obliquus oligosaccharide obtained in the previous step $\longrightarrow$ column chromatography with DEAE-cellulose $\longrightarrow$ Inonotus obliquus oligosaccharide IOP-2 $\longrightarrow$ column chromatography with Sepharose G-200 $\longrightarrow$ Inonotus obliquus oligosaccharide IOP-2A $\longrightarrow$ dialysis $\longrightarrow$ vacuum concentration $\longrightarrow$ freeze-drying $\longrightarrow$ purified oligosaccharide IOP-2A. The content of IOP-2 in each purified component of Inonotus obliquus oligosaccharide was the largest. IOP-2A and IOP-2B were obtained after Sepharose G-200 column chromatography. Two purified components were obtained: IOP-2A is purified oligosaccharide and IOP-2B is oligosaccharide glycoprotein. We mainly carried out the structure and lipid-lowering test of IOP-2A.

2.4. Determination of the IOP-2A Infrared Spectrum. Six kinds of purified Inonotus obliquus oligosaccharides were obtained. The preliminary experiment of reducing blood lipid in mice was carried out. There was no significant difference in the lipid-lowering activity of each oligosaccharide component. Therefore, next, we mainly study the oligosaccharide IOP-2A with high content. $2 \mathrm{mg}$ Inonotus obliquus oligosaccharide IOP-2A was weighed. $200 \mathrm{mg} \mathrm{KBr}$ was added. $\mathrm{KBr}$ was ovendried to constant weight at $105^{\circ} \mathrm{C}$ before use. The oligosaccharide was mixed with $\mathrm{KBr}$ for grinding and tablet pressing. The determination was carried out with an infrared spectrometer in the range of wavenumber $400-4000 \mathrm{~cm}^{-1}$.

2.5. Determination of Molecular Weight of IOP-2A Inonotus obliquus Oligosaccharide. The molecular weight determination was done by high-performance gel permeation chromatography, with an RID-10A parallax refraction detector, Shimadzu CLASS-VP workstation, and TSKgel G-3000PWXL stainless steel chromatographic column $(7.8 \times 300 \mathrm{~mm})$. The column temperature was $35^{\circ} \mathrm{C}$. The concentration of the oligosaccharide sample was $5 \mathrm{mg} / \mathrm{ml}$, and the loading amount was $20 \mu \mathrm{L}$. The mobile phase was $0.2 \mathrm{M} \mathrm{NaCl}$ aqueous solution with a flow rate of $0.6 \mathrm{~mL} / \mathrm{min}$.

2.6. Determination of Inonotus obliquus Oligosaccharide by Using a Scanning Electron Microscope. The oligosaccharide of Inonotus obliquus was observed by using a scanning electron microscope. The sample, surface morphology, structure, size, and other information can be obtained directly. Oligosaccharide samples need to be sprayed with gold before observation.

2.7. Determination of the Oligosaccharide Composition of Inonotus obliquus. Weigh $5 \mathrm{mg}$ of purified Inonotus obliquus oligosaccharide IOP-2A, and add $5 \mathrm{ml} 2 \mathrm{M}$ trichloroacetic acid solution. The mixture was kept at $120^{\circ} \mathrm{C}$ for $2 \mathrm{~h}$ to the oligosaccharide which was hydrolyzed, and the hydrolysate was used for standby.

Mix monosaccharide standard solution, and prepare $20 \mathrm{mg} / \mathrm{ml}$ concentrated solution, respectively. Then, mix and dilute into mixed monosaccharide standard solutions of $0.5 \mathrm{mg} / \mathrm{ml}, 1.0 \mathrm{mg} / \mathrm{ml}, 1.5 \mathrm{mg} / \mathrm{mL}, 2.0 \mathrm{mg} / \mathrm{mL}, 2.5 \mathrm{mg} / \mathrm{mL}$, and $3.0 \mathrm{mg} / \mathrm{mL}$.

Derivatization of 1-phenyl-3-methyl-5-pyrazolone: $200 \mu \mathrm{L}, 0.5 \mathrm{M}$ sodium hydroxide solution and 1-phenyl-3methyl-5-pyrazolone methanol solution were added, 
respectively. Nitrogen was added to the mixture and it was covered and kept at $70^{\circ} \mathrm{C}$ for $30 \mathrm{~min}$. Then, the mixture was added to $200 \mathrm{~mL}$ hydrochloric acid solution with concentration of $0.5 \mathrm{M}$ to make the $\mathrm{pH}$ value of the sample about 7 . Add $1 \mathrm{~mL}$ of chloroform, and extract for more than three times. The water intake layer sample is analyzed by the liquid phase. Take $200 \mu \mathrm{L}$ mixed monosaccharide standard solution and analyze by liquid chromatography according to the same method.

The liquid chromatographic conditions were Shimadzu HPLC System (LC-10ATvp pump and SPD-10AVD UV detector) and COSMOSIL 5C18-PAQ column $(4.6 \times 250 \mathrm{~mm})$. The mobile phase was PBS $(0.1 \mathrm{M}, \mathrm{pH} 7.0)$ : acetonitrile $=81: 19(\mathrm{~V} / \mathrm{V})$. The flow rate was $1.0 \mathrm{~mL} / \mathrm{min}$, and the injection volume was $10 \mu \mathrm{L}$. The detection wavelength was $245 \mathrm{~nm}$.

2.8. Structure Setting Test of Inonotus obliquus Oligosaccharide. The methylation method was used. Analysis by GC-MS: the final methylation product was thoroughly dried and dissolved with $1 \mathrm{ml}$ of dichloromethane. After membrane filtration, GC-MS analysis was performed. Agilent Technologies 7890B GC and 5977B MSD systems were used for GC-MS analysis. The chromatographic column is Agilent DB- $35 \mathrm{~ms}$. Temperature rise procedure: $140^{\circ} \mathrm{C}$ for $2 \mathrm{~min}$. At $5^{\circ} \mathrm{C} / \mathrm{min}$, raise the temperature to $170^{\circ} \mathrm{C}$ and keep it for $3 \mathrm{~min}$. At $1^{\circ} \mathrm{C} / \mathrm{min}$, raise the temperature to $180^{\circ} \mathrm{C}$ and keep it for $5 \mathrm{~min}$. At $3^{\circ} \mathrm{C} / \mathrm{min}$, raise the temperature to $220^{\circ} \mathrm{C}$ and keep it for $1 \mathrm{~min}$. Raise the temperature to $295^{\circ} \mathrm{C}$ at $20^{\circ} \mathrm{C} / \mathrm{min}$ and keep it for $3 \mathrm{~min}$.

2.9. Mouse Test. KM mice were fed in isolation for one week. Then, they were randomly divided into 4 groups, as shown in Table 1 . They were allowed to eat and drink freely. Inonotus obliquus oligosaccharide IOP-2A was administered by gavage.

The mice were weighed once a week. To study the effect of Inonotus obliquus oligosaccharide on the body weight of mice, before the end of the gavage oligosaccharide experiment, they were fasting and drank water freely for $12 \mathrm{~h}$. Blood was collected from the mouse orbit. The blood was centrifuged at $4^{\circ} \mathrm{C}$ and $2158 \times \mathrm{g}$ for $15 \mathrm{~min}$. The serum obtained by centrifugation was stored in a refrigerator at $-20^{\circ} \mathrm{C}$. It was used for the determination of blood indexes. The mice, after blood collection, were killed by spinal dislocation. The liver and kidney were taken. After weighing the liver, take $0.1 \mathrm{~g}$ and place it in a centrifuge tube. It was used for the determination of SOD and MDA. Wash with normal saline, and dry the surface with filter paper. Place in a centrifuge tube and weigh. Liver index and kidney index were calculated. TC, TG, LDL-C, and HDL-C were determined in mouse serum. The contents of TC, TG, LDL-C, and HDL-C in mouse serum were determined by kits. SOD and MDA were determined in the mouse liver.

The frozen mouse liver was added with normal saline in the ratio of $1: 9$ to make a homogenate with a concentration of $10 \%$. The blood was centrifuged at $4^{\circ} \mathrm{C}$ and $2158 \times \mathrm{g}$ for 15 min. The supernatant was taken, and the values of SOD and
MDA were measured. The instructions of the kit were followed.

2.10. Study on the Mechanism of IOP-2A Reducing Blood Lipid. The mechanism of oligosaccharide reducing blood lipid was studied from the changes of intestinal flora in mice. Mouse feces were taken from the superclean workbench. They were put in the frozen storage tube and were put in liquid nitrogen for storage. Microbial DNA was extracted. DNA purity and concentration were measured. The fecal flora of mice was determined by high-throughput sequencing. The variable region $\mathrm{V} 4$ of the $16 \mathrm{~S}$ rDNA gene was amplified. The primers of the bacterial $16 \mathrm{~S}$ rDNA V4 region are shown in Table 2.

Reaction system of PCR: Phusion Master Mix $15 \mu \mathrm{L}$, $2 \mu \mathrm{M}$ Primer $3 \mu \mathrm{L}, 1 \mathrm{ng} / \mu \mathrm{Lg}$ DNA $10 \mu \mathrm{L}$, and $\mathrm{H}_{2} \mathrm{O} 2 \mu \mathrm{L}$. Reaction procedure: $98^{\circ} \mathrm{C}, 10 \mathrm{sec} ; 50^{\circ} \mathrm{C}, 30 \mathrm{sec}$; and $72^{\circ} \mathrm{C}$, 5 min.

The products were separated by $2 \%$ agarose gel electrophoresis. The target strips were recycled.

They were purified using the GeneJET kit of Thermo Scientific. The library was constructed using the TruSeq DNA PCR-free library preparation kit of Illumina company. After the library is tested to be qualified, PCR amplification products were sequenced by computer. The sequenced data were spliced and filtered. Valid data were obtained. The richness, diversity, and species composition of OTUs (operational taxonomic units) were analyzed based on effective data.

2.11. Test Data Processing and Analysis. The detection indexes were measured for three times, and the average value was taken. The test results are expressed as "mean \pm standard deviation." SPSS software was used for data processing.

\section{Results and Discussion}

3.1. Determination Results of the IOP-2A Infrared Spectrum. According to the infrared spectrum, the wavenumber 1000-1200 $\mathrm{cm}^{-1}$ corresponds to the telescopic vibration absorption peak of C-O-C. The wavenumber $1300-1500 \mathrm{~cm}^{-1}$ corresponds to the $\mathrm{C}-\mathrm{H}$ deformation vibration absorption peak. Wavenumber $1600 \mathrm{~cm}^{-1}$ corresponds to the $\mathrm{C}=\mathrm{O}$ bond structure, and wavenumber $2900 \mathrm{~cm}^{-1}$ corresponds to the $\mathrm{C}-\mathrm{H}$ stretching vibration absorption peak. The wavenumber around $3400 \mathrm{~cm}^{-1}$ corresponds to the $\mathrm{O}-\mathrm{H}$ stretching vibration absorption peak. There is a strong absorption peak near the wavenumber of $880 \mathrm{~cm}^{-1}$. Therefore, it can be seen that the glycosidic bond of IOP-2A is mainly $\beta$-type glycosidic bond. See Figure 1.

3.2. Scanning Electron Microscope Measurement Results of $I O P-2 A$. See Figure 2 for the measurement results of Inonotus obliquus oligosaccharide IOP-2A by using the scanning electron microscope. It can be seen from the figure that Inonotus obliquus oligosaccharide macromolecules form a layered structure. 
TABLE 1: Groups of mice.

\begin{tabular}{lccc}
\hline Group & Number & Feed type & Administration \\
\hline Normal group (N) & 10 & Common feed & Equal amount of distilled water \\
High-fat model group (M) & 10 & High-fat feed & Equal amount of distilled water \\
IOP-2A high-dose group (H) & 10 & High-fat feed & $400 \mathrm{mg} / \mathrm{kg} \mathrm{bw}$ \\
IOP-2A low-dose group (L) & 10 & High-fat feed & $200 \mathrm{mg} / \mathrm{kg} \mathrm{bw}$ \\
\hline
\end{tabular}

3.3. Determination Results of Molecular Weight and Composition of IOP-2A. The molecular weight of oligosaccharides was measured by high-performance gel permeation chromatography. The results show that $\mathrm{Mn}$ of IOP-2A is about $1000 \mathrm{Da}$, as shown in Figure 3. The monosaccharide composition and molar ratio of IOP-2A are glucose : xylose : galactose $:$ mannose $=54.1: 13.6: 13.2: 6.7$. In addition, it also contains a small amount of galactose, gluconic acid, rhamnose, and fucose. See Figure 4.

3.4. Methylation Analysis Results of Inonotus obliquus Oligosaccharide IOP-2A. After complete methylation, acid hydrolysis, reduction, and acetylation of the sample, methyl sugar alcohol acetate derivatives were obtained. The glycosidic linkage was analyzed by GC-MS. Figure 5 is the separation chromatogram of oligosaccharide. The mass spectrum of each peak in the chromatogram is analyzed. Compared with the standard map, the types of glycosyl linkages in the sample are summarized in Table 3. The ion fragment peaks corresponding to each peak are shown in Figure 6.

3.5. Results of the Mouse Weight Test. The mice were weighed once a week. To study the effect of Inonotus obliquus oligosaccharide on the body weight of KM mice, the weight value of mice in each group and the change trend of weight with time are shown in Figure 7. The measurement results of liver and kidney indexes of mice are shown in Figure 6. The data showed that the liver index of the high-fat model group increased significantly compared with the blank control group due to the intake of high-fat diet. The determination results of TC, TG, LDL-C, and HDL-C in serum of KM mice are shown in Table 4. See Table 5 for the determination results of SOD and MDA contents in the liver of KM mice. According to the data, Inonotus obliquus oligosaccharide IOP-2A has obvious lipid-lowering effect and has a significant dose-response relationship.

3.6. Measurement Results of Liver and Kidney Indexes of KM Mice. See Figure 8. It can be seen from the figure that IOP-2A can make the liver index and kidney index of mice close to normal values, and there is an obvious dose-effect relationship.

3.7. Determination Results of TC, TG, LDL-C, and HDL-C in Mouse Serum. See Table 4. According to the data, IOP-2A can prevent hyperlipidemia and has an obvious dose-response relationship.
TABle 2: Primers of the bacterial $16 \mathrm{~S}$ rDNA V4 region.

\begin{tabular}{lccc}
\hline Type & Region & $\begin{array}{c}\text { Primer } \\
\text { name }\end{array}$ & Primer sequence \\
\hline Bacterial & \multirow{2}{*}{ V4 } & 515F & GTGCCAGCMGCCGCGGTAA \\
$16 \mathrm{~S}$ & & $806 \mathrm{R}$ & GGACTACHVGGGTWTCTAAT \\
\hline
\end{tabular}

3.8. Determination Results of SOD and MDA Contents in the Mouse Liver. See Table 5. According to the data, IOP-2A can protect the liver and has an obvious dose-response relationship.

3.9. Differences in the Out Level of Fecal Flora in Mice. The mechanism of reducing blood lipid by oligosaccharide was studied from the changes of intestinal flora in mice. Mouse feces were taken from the superclean workbench and placed in the frozen storage tube. They were kept in liquid nitrogen for standby. Microbial DNA was extracted, and its purity and concentration were measured. The fecal flora of mice was determined by high-throughput sequencing. The differences of out in each group were determined according to gene $97 \%$ similarity. There were 1327 kinds of bacteria in the fecal flora of mice in the normal group (group N), highfat model group (group M), high-dose IOP-2A group (group $\mathrm{H}$ ), and low-dose IOP-2A group (group L). The out species of the IOP-2A high-dose group (group $\mathrm{H}$ ) were 888 , accounting for $66.92 \%$, and the abundance was the highest. IOP-2A high-dose group (group $\mathrm{H}$ ) $>$ IOP-2A low-dose group (group L) > normal group (group N) > high-fat model group (group M), as shown in Figure 9.

3.10. Alpha Diversity Analysis of Mouse Fecal Flora. For alpha diversity, observed species diversity index was selected. It can represent the diversity and uniformity of species distribution and can show the depth and amount of data of sequencing. The diversity index curves of the hyperlipidemia prevention blank group (group N), hyperlipidemia model group (group M), IOP-2A high-dose group (group H), and IOP-2A low-dose group (group L) are shown in Figure 10.

3.11. Beta Diversity Analysis of Mouse Fecal Flora. The contribution rate of the first principal component of fecal flora of KM mice to the sample difference was $57.58 \%$. The contribution rate of the second principal component to the sample difference was $16.37 \%$. The IOP-2A high-dose group (group $\mathrm{H}$ ) and IOP-2A low-dose group (group L) have a strong correlation, as shown in Figure 11. 


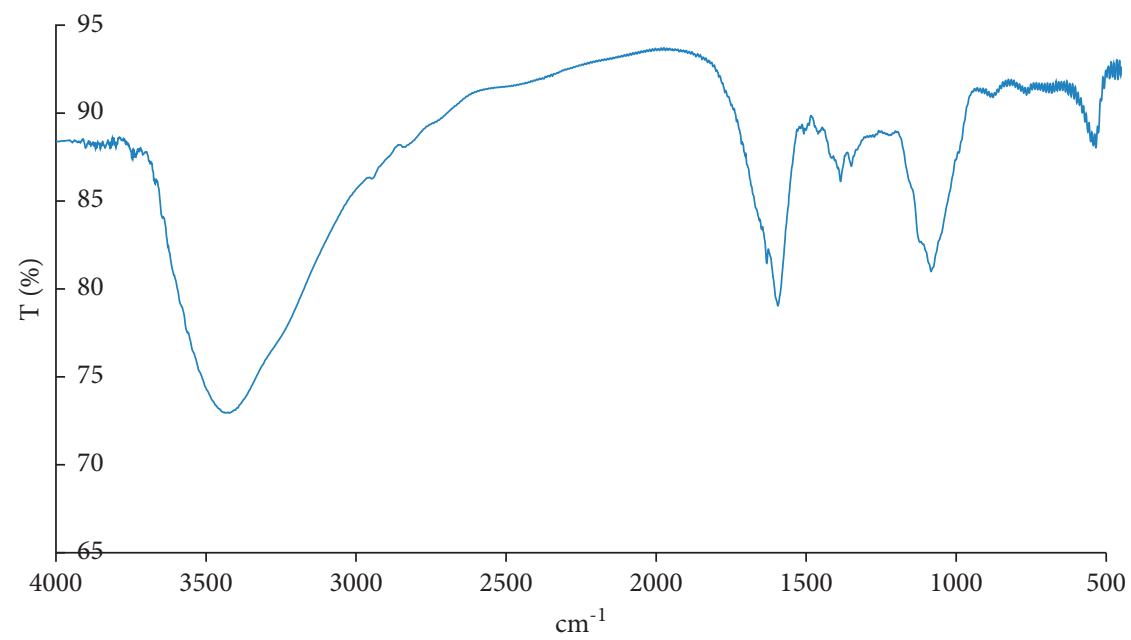

FIGURE 1: Infrared spectrum determination curve of Inonotus obliquus oligosaccharide IOP-2A.
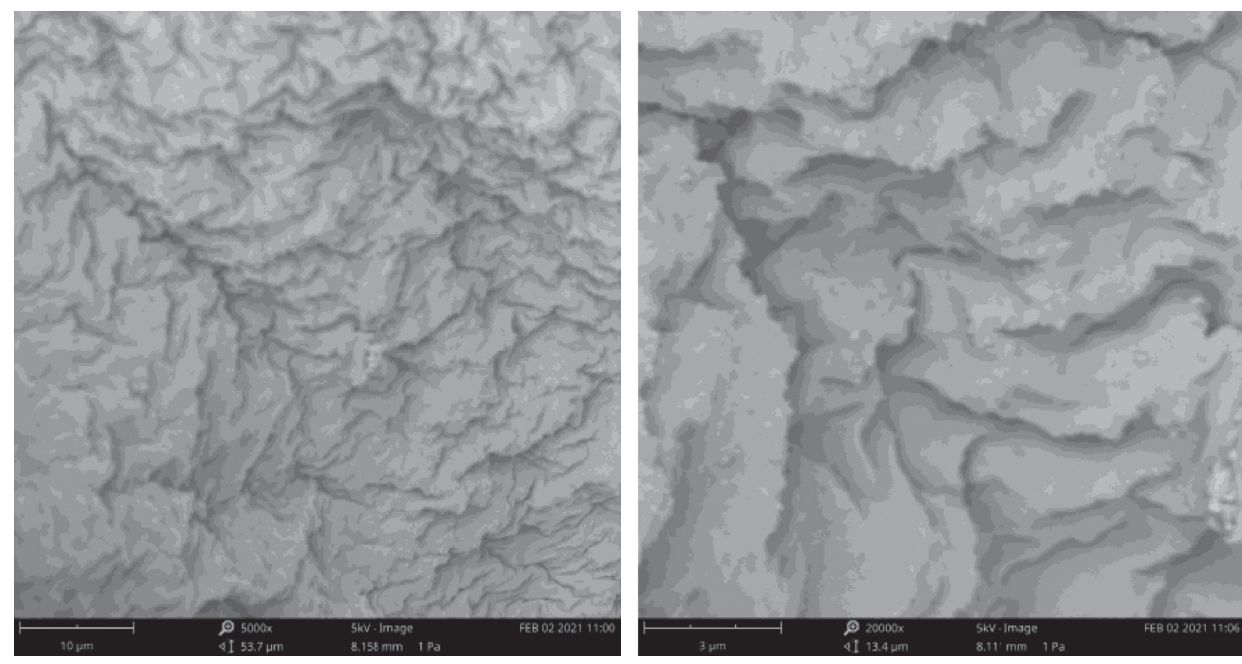

FIGURE 2: Scanning electron microscope observation of Inonotus obliquus-purified oligosaccharide IOP-2A.

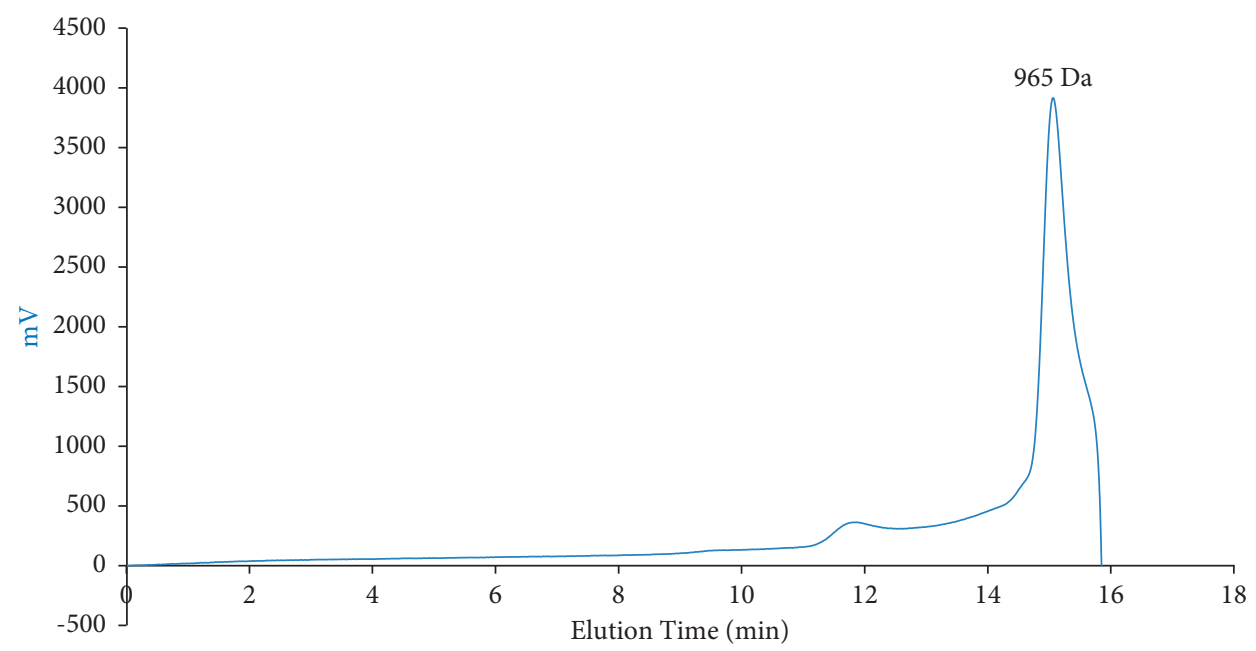

Figure 3: Molecular weight determination curve of Inonotus obliquus oligosaccharide IOP-2A. 


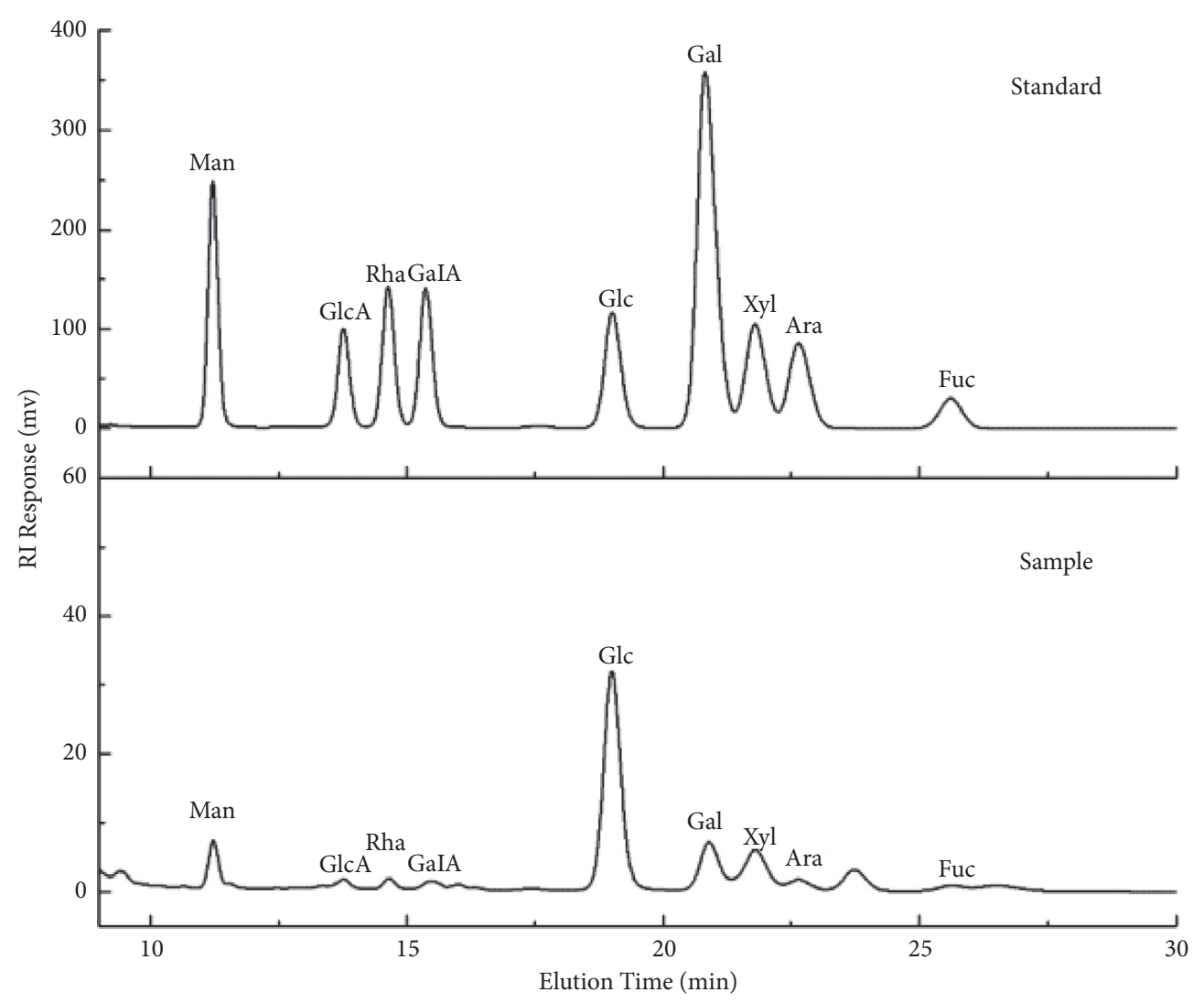

FIgURE 4: Composition of IOP-2A monosaccharide and liquid phase curve of the standard.

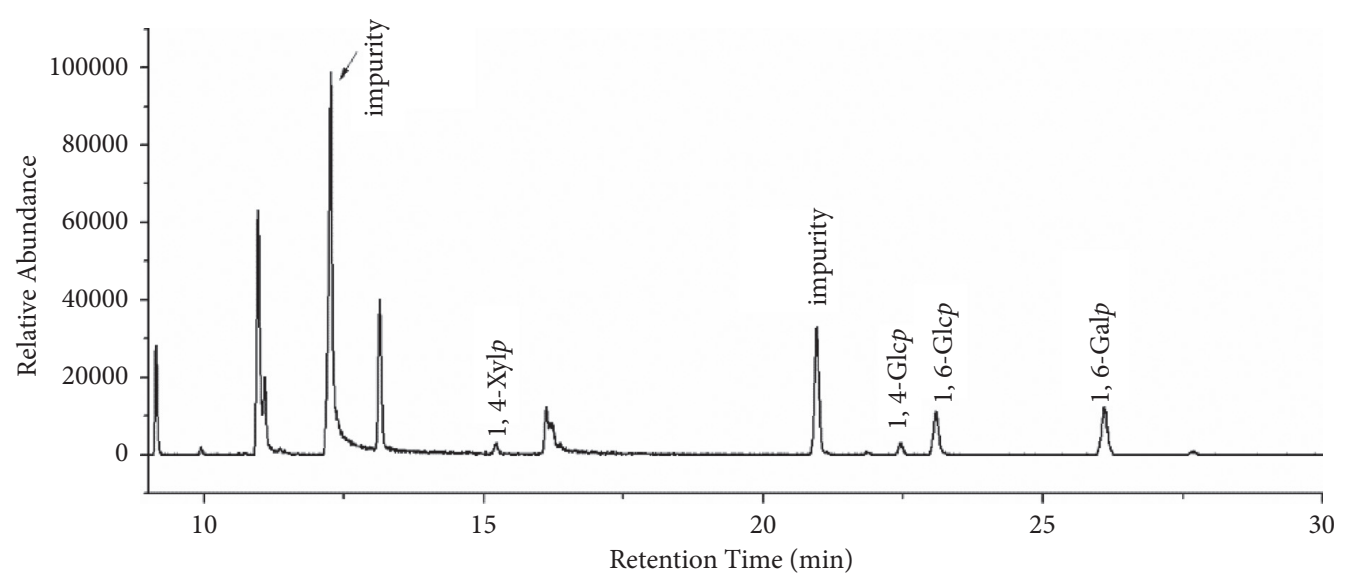

FIGURE 5: GC-MS TIC of Inonotus obliquus oligosaccharide IOP-2A.

TABLE 3: Glycosidic bond connection mode of Inonotus obliquus oligosaccharide IOP-2A.

\begin{tabular}{lccc}
\hline PMAA & Connection mode & Percentage (mol\%) & Fragment ion peak (M/z) \\
\hline $2,3,6-\mathrm{Me}_{3}-\mathrm{Glcp}$ & $1,4-$ & 9.2 & $87,101,117,129,233$ \\
$2,3,4-\mathrm{Me}_{3}-\mathrm{Glcp}$ & $1,6-$ & 41.7 & $101,117,129,161,173,189,233$ \\
$2,3,4-\mathrm{Me}_{3}-\mathrm{Galp}$ & $1,6-$ & 43.4 & $87,101,117,129,161,173,189,233$ \\
\hline
\end{tabular}

3.12. Diversity Analysis at the Phylum Level. More than 1300 kinds of microorganisms were detected by gene sequencing analysis of mouse fecal flora. The following is an analysis according to the classification order of phyla, class, order, family, genus, and species. The top 10 in the gate level are as follows: Bacteroidetes, Firmicutes, Verrucomicrobia, Proteobacteria, Actinobacteria, Desulfobacterota, unidentified bacteria, Deferribacteres, Campilobacterota, and 

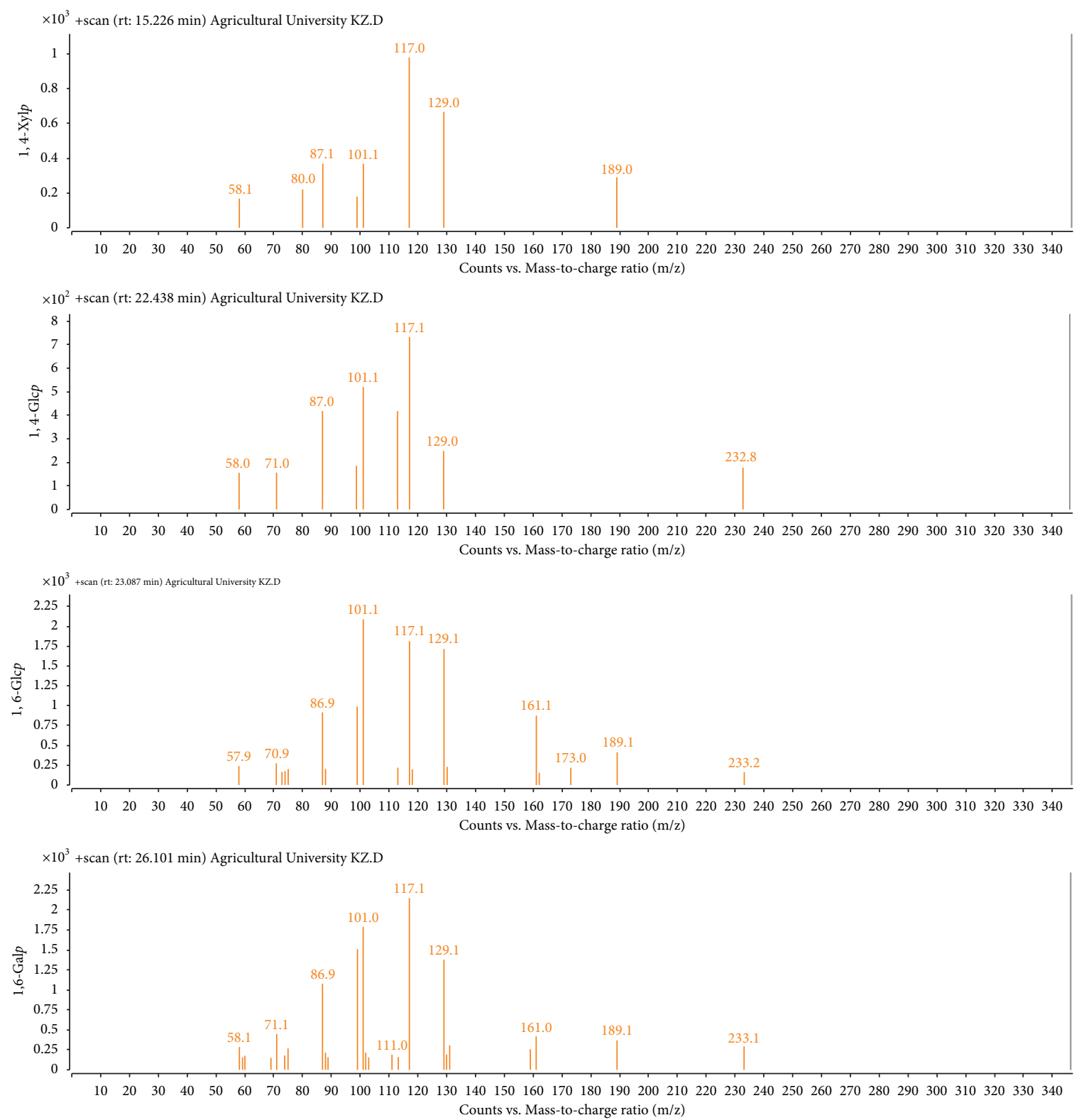

Figure 6: Ion fragments corresponding to each peak.

Acidobacteria. The diversity analysis at the gate level is shown in Figure 12. There were significant differences in flora among groups. The increased ratio of Firmicutes to Bacteroidetes can produce more fatty acids and increase blood lipids. It can be seen from Figure 10 that the ratio of Firmicutes/Bacteroidetes in the high-fat model group (M) increased [33, 34]. This causes an increase in blood lipids. The composition of flora in groups $\mathrm{L}$ and $\mathrm{N}$ was close, and the blood lipid level was close to the normal value. The ratio of Firmicutes to Bacteroidetes in group $\mathrm{H}$ deviated from the normal value. Moreover, the blood lipid level is close to the normal value, indicating that the amount of fat and bile adsorbed by IOP-2A increases. So, the absorption of fatty acids was reduced, and the rise of blood lipids was prevented by Inonotus obliquus oligosaccharide.
3.13. Diversity Analysis at the Order Level. The top 10 are as follows: Bacteroidales, Verrucomicrobiales, Erysipelotrichales, Burkholderiales, Lachnospirales, Lactobacillales, Coriobacteriales, Clostridia_UCG-014, Desulfovibrionales, and Clostridiales, where Clostridia_UCG-014 belongs to the order Priscilla, which can inhibit the increase of fat factors. The proportion of Clostridia_UCG-014 in the high-dose group of Inonotus obliquus oligosaccharide was higher than that of the low-dose group. There is a significant relationship between the amount and hypolipidemic effect. Bacteroidales belongs to the order of Bacteroides. The proportion of Bacteroidales in the high-dose group of Inonotus obliquus oligosaccharide is lower. Bacteroidales is negatively correlated with hypolipidemic effect [35]. It is consistent with the high-dose oligosaccharide group. See Figure 13. 


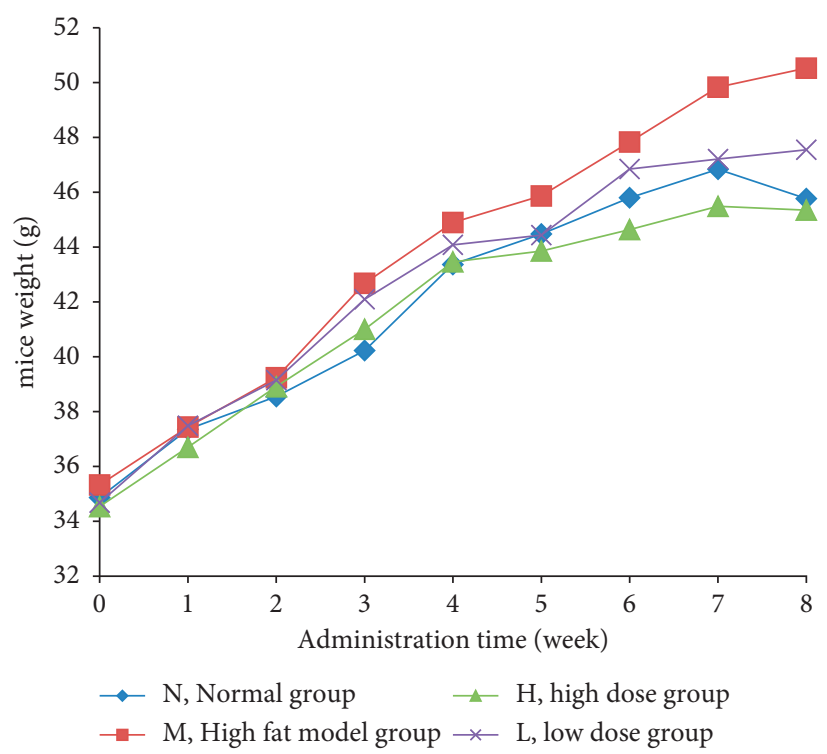

Figure 7: Variation trend of body weight of KM mice with time.

TABle 4: Determination results of components in serum of KM mice in each group.

\begin{tabular}{lcccc}
\hline Group & TC $(\mathrm{mmol} / \mathrm{L})$ & TG $(\mathrm{mmol} / \mathrm{L})$ & LDL-C $(\mathrm{mmol} / \mathrm{L})$ & HDL-C $(\mathrm{mmol} / \mathrm{L})$ \\
\hline Normal group $(\mathrm{N})$ & $2.984 \pm 0.209$ & $1.538 \pm 0.135$ & $0.215 \pm 0.018$ & $2.383 \pm 0.164$ \\
High-fat model group $(\mathrm{M})$ & $4.447 \pm 0.396$ & $2.535 \pm 0.200$ & $0.645 \pm 0.081$ & $1.494 \pm 0.127$ \\
High-dose group (H) & $3.237 \pm 0.256^{*}$ & $1.616 \pm 1.277^{*}$ & $0.269 \pm 0.020^{*}$ & $2.047 \pm 0.182^{*}$ \\
Low-dose group (L) & $3.406 \pm 0.221^{*}$ & $1.957 \pm 0.153^{*}$ & $0.323 \pm 0.026^{*}$ & $1.836 \pm 0.147^{*}$ \\
\hline
\end{tabular}

Note: compared with the model group, ${ }^{*} P<0.05$.

TABLE 5: Determination results of SOD and MDA in the liver of KM mice in each group.

\begin{tabular}{lcc}
\hline Group & SOD (U/mg prot) & MDA (nmol/mg prot) \\
\hline Normal group (N) & $363.13 \pm 27.60$ & $2.21 \pm 0.16$ \\
High-fat model group (M) & $198.54 \pm 17.47$ & $4.91 \pm 0.41$ \\
High-dose group (H) & $317.47 \pm 20.00^{*}$ & $2.89 \pm 0.23^{*}$ \\
Low-dose group (L) & $279.15 \pm 21.22^{*}$ & $3.41 \pm 0.25^{*}$ \\
\hline
\end{tabular}

Note: compared with the model group, ${ }^{*} P<0.05$ or alpha diversity, observed species diversity ind.

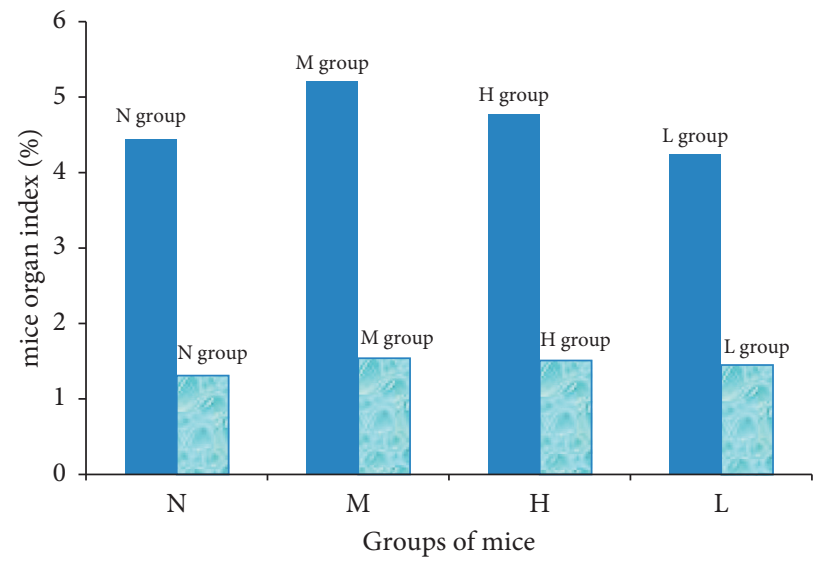

- Liver index

$\square$ Renal index

FIGURE 8: Organ index of KM mice in each group. 


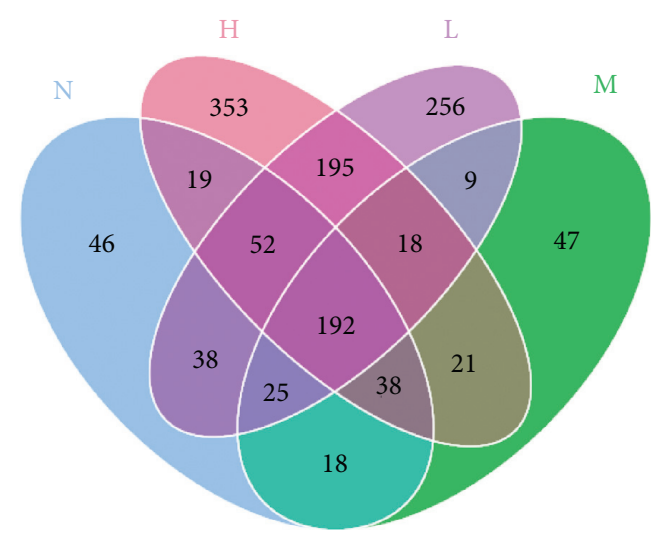

FIgURE 9: Venn diagram of each group.

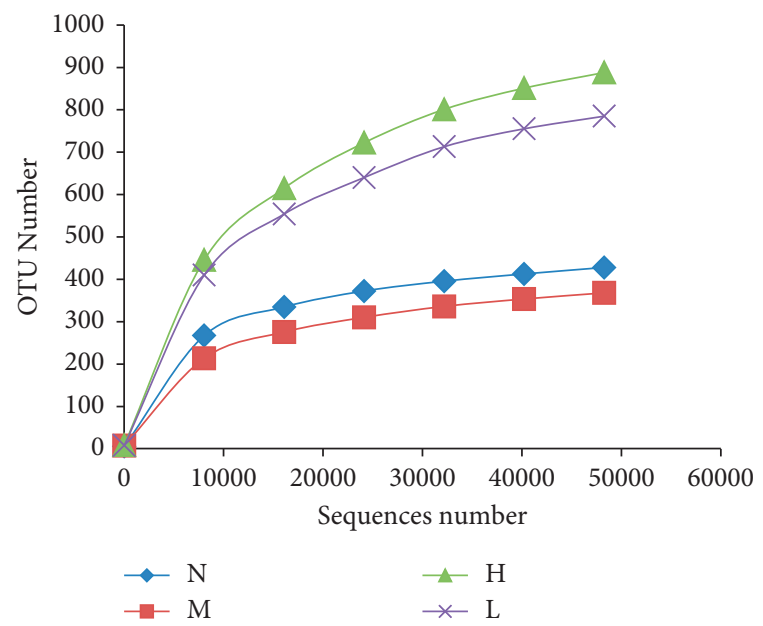

FIGURE 10: Number of species in each group.

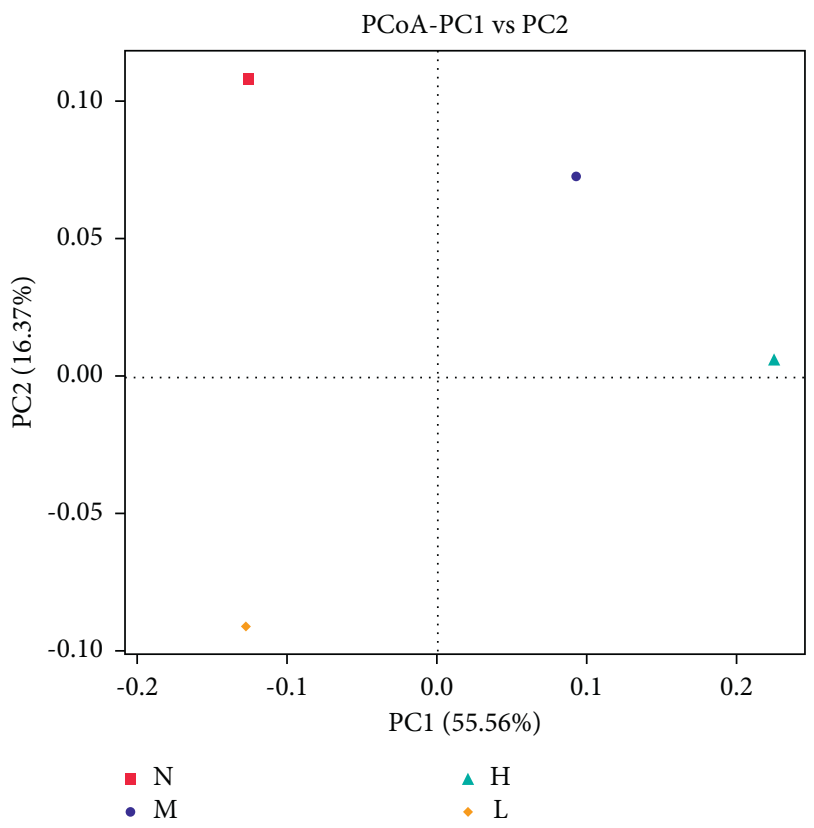

Figure 11: PCoA of each group.

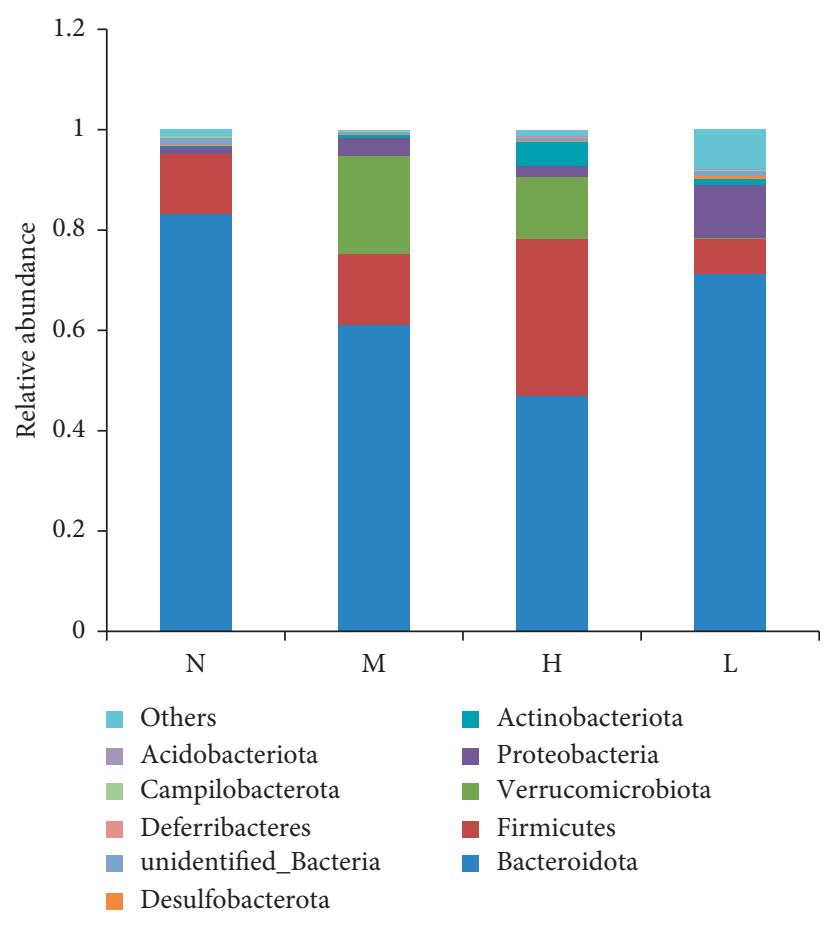

Figure 12: Relative abundance at the phylum level.

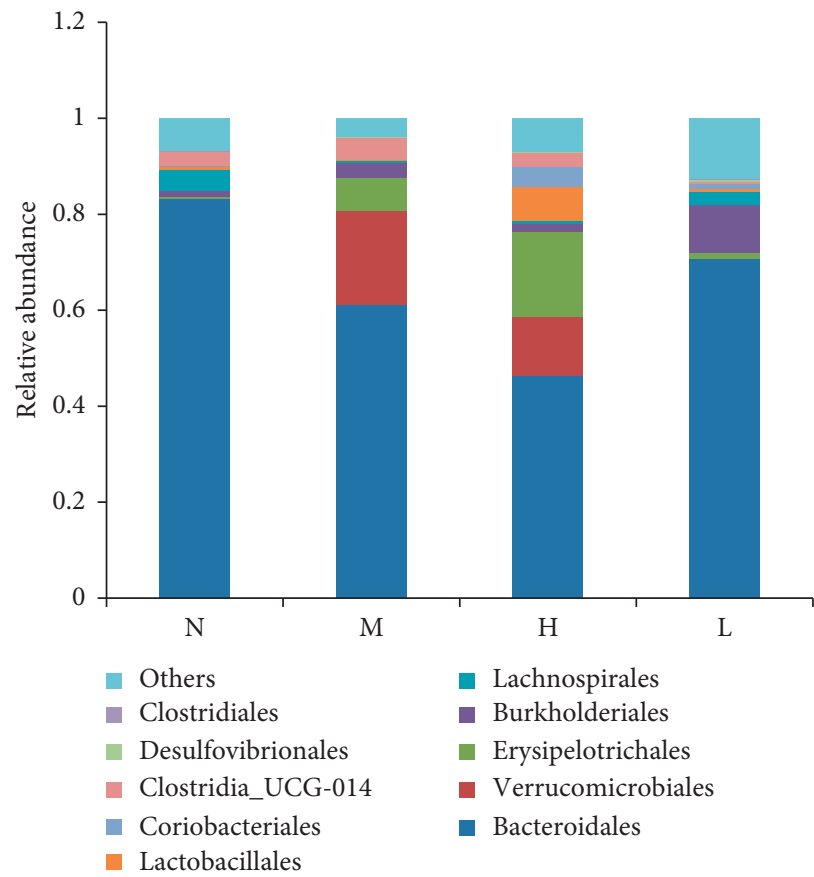

FIGURE 13: Relative abundance at the order level.

\section{Conclusion}

The molecular weight $\mathrm{Mw}$ of Inonotus obliquus oligosaccharide IOP-2A extracted by hot water was about $1 \mathrm{kDa}$. The main monosaccharide composition and molar ratio of Inonotus obliquus oligosaccharide IOP-2A were glucose: xylose $:$ galactose $:$ mannose $=54.1: 13.6: 13.2: 6.7$. In addition, there was a small amount of arabinose, galactic acid, 
gluconic acid, rhamnose, and fucose. The glycosidic bond between monosaccharide molecules was $\beta$-type glycosidic bond. Oligosaccharide macromolecules formed a layered structure. IOP-2A had the function of preventing hyperlipidemia. The mechanism of IOP-2A in preventing hyperlipidemia was to reduce the intake of fatty acids and other nutrients by improving the balance of intestinal flora in mice, so as to realize the role of preventing hyperlipidemia. Inonotus obliquus oligosaccharide IOP-2A can be used as a functional food component to prevent hyperlipidemia, which has certain theoretical and application value. Our research team considers the further mechanism of Inonotus obliquus oligosaccharide in the prevention of hyperlipidemia.

\section{Data Availability}

The data used to support the findings of this study are included within the article.

\section{Conflicts of Interest}

The authors declare that there are no conflicts of interest regarding the publication of this article.

\section{Acknowledgments}

This research was supported by the National Key Research and Development Project "Efficient preparation of functional components of edible fungi and innovative product development and industrialization demonstration" (Project no. 2018yfd0400204).

\section{References}

[1] A. M. Agha and C. M. Ballantyne, "Management of hyperlipidaemia among statin-intolerant patients after acute coronary syndrome: where do we stand in 2020?" European journal of preventive cardiology, vol. 28, no. 1, pp. 30-32, 2021.

[2] Y. Nakashima, "Treatment of hyperlipidemia," Internal Medicine, vol. 39, no. 10, pp. 765-772, 2000.

[3] X. C. Wang, J. H. Pan, Z. P. Ren et al., "Application of a novel hybrid algorithm of Bayesian network in the study of hyperlipidemia related factors: a cross-sectional study," $B M C$ Public Health, vol. 21, no. 1, Article ID 1375, 2021.

[4] E. Zhou, H. Geerte, L. Zhuang et al., "Colesevelam enhances the beneficial effects of brown fat activation on hyperlipidaemia and atherosclerosis development," Cardiovascular Research, vol. 116, no. 10, pp. 1710-1720, 2020.

[5] B. Holven Kirsten and M. Ulven Stine, "Bogsrud Martin P,Hyperlipidaemia and cardiovascular disease and impact of early cholesterol accumulation," Current Opinion in Lipidology, vol. 30, no. 6, pp. 490-493, 2013.

[6] W. Mayumi, A. Oh, O. Yoshie, F. Guelfucci, Y. Shimasaki, and T. Teramoto, "Adherence and persistence to hyperlipidemia medications in patients with atherosclerotic cardiovascular disease and those with diabetes mellitus based on administrative claims data in Japan," Atherosclerosis, vol. 282, pp. 19-28, 2018.

[7] B. Camelia, F. Andreea, D. Leucuta, M. Cristina, M. Bojita, and D. L. Dumitrascu, "Drug-drug interactions of statins potentially leading to muscle-related side effects in hospitalized patients," Romanian Journal of Internal Medicine, vol. 53, no. 4, pp. 329-335, 2015.

[8] T. Berent, R. Berent, S Steiner, and H. Sinzinger, "Statininduced muscular side effects at rest and exercise-an anatomical mapping," Atherosclerosis Supplements, vol. 40, pp. 73-78, 2019.

[9] D. G. Karalis, R. A. Wild, K. C. Maki et al., "Gender differences in side effects and attitudes regarding statin use in the Understanding Statin Use in America and Gaps in Patient Education (USAGE) study," Journal of Clinical Lipidology, vol. 10, no. 4, pp. 833-841, 2016.

[10] S. Govindan, A. Jayabal, J. Shanmugam, and P. Ramani, "Antioxidant and hepatoprotective effects of Hypsizygus ulmarius polysaccharide on alcoholic liver injury in rats," Food Science and Human Wellness, vol. 10, no. 4, pp. 523-535, 2021.

[11] Z. Yin, Z. Liang, C. Li, J. Wang, C. Ma, and W. Kang, "Immunomodulatory effects of polysaccharides from edible fungus: a review," Food Science and Human Wellness, vol. 10, no. 4, pp. 393-400, 2021.

[12] Y. Zhang, D. Wang, Y. Chen et al., "Healthy function and high valued utilization of edible fungi," Food Science and Human Wellness, vol. 10, no. 4, pp. 408-420, 2021.

[13] X. Liu, Z. Ren, R. Yu et al., "Structural characterization of enzymatic modification of Hericium erinaceus polysaccharide and its immune-enhancement activity," International Journal of Biological Macromolecules, vol. 166, pp. 1396-1408, 2021.

[14] S. S. Zhang, S. P. Nie, D. F. Huang, J. Huang, Y. Feng, and M. Xie, "A polysaccharide from ganoderma atrum inhibits tumor growth by induction of apoptosis and activation of immune response in CT26-bearing mice," Agricultural and Food Chemistry, vol. 62, no. 35, pp. 9296-9304, 2014.

[15] K.-H. Wong, C. K. M. Lai, and P. C. K. Cheung, "Immunomodulatory activities of mushroom sclerotial polysaccharides," Food Hydrocolloids, vol. 25, no. 2, pp. 150-158, 2011.

[16] X. Du, H. Mu, S. Zhou, Y. Zhang, and X. Zhu, "Chemical analysis and antioxidant activity of polysaccharides extracted from Inonotus obliquus sclerotia," International Journal of Biological Macromolecules, vol. 62, pp. 691-696, 2013.

[17] J. Y. Kim, K. D. Moon, and H. I. Kang, “Antioxidative and antitumor activities of crude polysaccharide fraction from pleurotus eryngii," Journal of The Korean Society of Food Science and Nutrition, vol. 33, no. 10, pp. 1589-1593, 2021.

[18] L. Fan, S. Ding, L. Ai, and K. Deng, "Antitumor and immunomodulatory activity of water-soluble polysaccharide from Inonotus obliquus," Carbohydrate Polymers, vol. 90, no. 2, pp. 870-874, 2012.

[19] H.-H. Sun, W.-J. Mao, Y. Chen et al., "Isolation, chemical characteristics and antioxidant properties of the polysaccharides from marine fungus Penicillium sp. F23-2," Carbohydrate Polymers, vol. 78, no. 1, pp. 117-124, 2009.

[20] E. Vamanu, "Antioxidant properties of polysaccharides obtained by batch cultivation of Pleurotus ostreatusmycelium," Natural Product Research, vol. 27, no. 12, pp. 1115-1118, 2013.

[21] E.-S. Lin, "Production of exo polysaccharides by submerged mycelial culture of Grifola frondosa TFRI1073 and their antioxidant and antiproliferative activities," World Journal of Microbiology and Biotechnology, vol. 27, no. 3, pp. 555-561, 2012.

[22] Y. J. Hwang, H. K. Nam, M. J. Chang, K.-W. Noh, and S.-H. Kim, "Effect of lentinus edodes and pleurotus eryngii extracts on proliferation and apoptosis in human colon cancer cell lines," Journal-of-the-Korean-Society-of-Food-Scienceand-Nutrition, vol. 32, no. 2, pp. 217-222, 2003. 
[23] X. Cao, R. Liu, J. Liu et al., "A novel polysaccharide from lentinus edodes mycelia exhibits potential antitumor activity on laryngeal squamous cancer cell line hep-2," Applied Biochemistry and Biotechnology, vol. 171, no. 6, pp. 1444-1453, 2013.

[24] S. Wasser, "Medicinal mushrooms as a source of antitumor and immunomodulating polysaccharides," Applied Microbiology and Biotechnology, vol. 60, no. 3, pp. 258-274, 2002.

[25] J. Shi, C. Cheng, H. Zhao, J. Jing, N. Gong, and W. Lu, "In vivo anti-radiation activities of the Ulva pertusa polysaccharides and polysaccharide-iron(III) complex," International Journal of Biological Macromolecules, vol. 60, pp. 341-346, 2013.

[26] W. Wang, C. Xue, and X. Mao, "Radioprotective effects and mechanisms of animal, plant and microbial polysaccharides," International Journal of Biological Macromolecules, vol. 153, pp. 373-384, 2020.

[27] I. Touiss, S. Khatib, O. Bekkouch, S. Amrani, and H. Harnafi, "Phenolic extract from Ocimum basilicum restores lipid metabolism in Triton WR-1339-induced hyperlipidemic mice and prevents lipoprotein-rich plasma oxidation," Food Science and Human Wellness, vol. 6, no. 1, pp. 28-33, 2017.

[28] D. Fang, D. Wang, G. Ma et al., "Auricularia polytricha noodles prevent hyperlipemia and modulate gut microbiota in high-fat diet fed mice," Food Science and Human Wellness, vol. 10, no. 4, pp. 431-441, 2021.

[29] T. L. Nguyen, J. Chen, Y. Hu et al., "In vitro antiviral activity of sulfated Auricularia auricula polysaccharides," Carbohydrate Polymers, vol. 90, no. 3, pp. 1254-1258, 2012.

[30] Y. Ye, D. Ji, L. You, L. Zhou, Z. Zhao, and C. Brennan, "Structural properties and protective effect of Sargassum fusiforme polysaccharides against ultraviolet B radiation in hairless Kun Ming mice," Journal of Functional Foods, vol. 43, pp. 8-16, 2018.

[31] C. Zhang, L. Zhang, H. Liu, J. Zhang, C. Hu, and L. Jia, "Antioxidation, anti-hyperglycaemia and renoprotective effects of extracellular polysaccharides from Pleurotus eryngii SI-04," International Journal of Biological Macromolecules, vol. 111, pp. 219-228, 2018.

[32] K. Zhu, S. Nie, C. Li et al., "A newly identified polysaccharide from Ganoderma atrum attenuates hyperglycemia and hyperlipidemia," International Journal of Biological Macromolecules, vol. 57, pp. 142-150, 2013.

[33] R. C. Bortolin, A. R. Vargas, J. Gasparotto et al., "A new animal diet based on human Western diet is a robust dietinduced obesity model: comparison to high-fat and cafeteria diets in term of metabolic and gut microbiota disruption," International Journal of Obesity, vol. 42, no. 3, pp. 525-534, 2018.

[34] B. Xue, J. Xie, J. Huang et al., "Plant polyphenols alter a pathway of energy metabolism by inhibiting fecal Bacteroidetes and Firmicutes in vitro," Food \& Function, vol. 7, no. 3, pp. 1501-1507, 2018.

[35] M. R. Clausen and P. B. Mortensen, "Kinetic studies on colonocyte metabolism of short chainfatty acids and glucose in ulcerative colitis," Gut, vol. 37, no. 5, pp. 684-689, 2018. 